\title{
One-Step Synthesis of Enhanced Band-Edge Emission ZnSe Nanowires Assisted by $\mathrm{SnO}_{2}$
}

\author{
Lipeng Hou ${ }^{\text {1*, }}$ Yongchang Guo ${ }^{2}$, Lijuan Zhang ${ }^{1}$ \\ ${ }^{1}$ School of Physics, Jinzhong University, Jinzhong, China \\ ${ }^{2}$ Beijing Key Laboratory of Nanophotonics and Ultrafine Optoelectronic Systems, School of Physics, Beijing Institute of \\ Technology, Beijing, China \\ Email: *houlipeng163@163.com
}

How to cite this paper: Hou, L.P., Guo, Y.C. and Zhang, L.J. (2020) One-Step Synthesis of Enhanced Band-Edge Emission $\mathrm{ZnSe}$ Nanowires Assisted by $\mathrm{SnO}_{2}$. Advances in Materials Physics and Chemistry, 10, 263-269.

https://doi.org/10.4236/ampc.2020.1011020

Received: October 9, 2020

Accepted: November 10, 2020

Published: November 13, 2020

Copyright (c) 2020 by author(s) and Scientific Research Publishing Inc. This work is licensed under the Creative Commons Attribution International License (CC BY 4.0).

http://creativecommons.org/licenses/by/4.0/

Open Access

\begin{abstract}
Zinc selenide $(\mathrm{ZnSe})$ is a direct band gap semiconductor material with a band gap of $2.7 \mathrm{eV}$ at room temperature, and is a very promising blue light emitting material. However, the ZnSe nanomaterials grown by the usual Au-catalyzed chemical vapor deposition (CVD) method are prone to cause defects in the material, and its luminescence spectra generally have strong yellow light emission, which seriously affects its application in blue light devices. The paper proposes a simple method for preparing blue light-emitting $\mathrm{ZnSe}$ nanowires. Adding a small amount of $\mathrm{SnO}_{2}$ to the source material $\mathrm{ZnSe}$ powder, when using the CVD method to grow ZnSe nanomaterials, can obtain $\mathrm{ZnSe}$ nanomaterials with only band edge blue emission. $\mathrm{SnO}_{2}$ can not only act as a catalyst, promote the growth of $\mathrm{ZnSe}$ nanowires and nanoribbons, reduce the growth temperature of nanomaterials, but also avoid the occurrence of deep level defects in ZnSe nanomaterials, and obtain pure blue light emitting $\mathrm{ZnSe}$ nanowires. The emission peak of the nanowire grown by this method can be shifted from 460 to $500 \mathrm{~nm}$. By precisely regulating the growth of the nanowire, it can make it emit light to cover the entire blue region, which can promote the application of inorganic semiconductor nanomaterials in the field of lighting and display.
\end{abstract}

\section{Keywords}

$\mathrm{ZnSe}, \mathrm{SnO}_{2}$, Photoluminescence, Blue Emission

\section{Introduction}

Zinc selenide (ZnSe) is an important II-VI semiconductor material with a direct band-gap of $\sim 2.7 \mathrm{eV}$ at room temperature, which is widely regarded as a very good candidate for blue-violet light emitting devices [1] [2] [3] [4]. With the 
miniaturization of devices, many research groups have begun to study the nanostructures of ZnSe materials and have done a lot of work in this area. In 2011, Liang et al. fabricated vertically aligned $\mathrm{ZnSe}$ nanowire arrays by metal-organic chemical vapor deposition (MOCVD) using $\mathrm{Ga}$ and $\mathrm{Ag}$ catalysts, while $\mathrm{SnO}_{2}$, it was found that there are strong deep level luminescence peaks in its photoluminescence (PL) spectra [5]. In 2014, Zannier et al. reported optimized photoluminescence of $\mathrm{ZnSe}$ nanowires grown by molecular beam epitaxy. Even with this expensive growth method, the deep-level defect emission peak in its PL spectrum is still significant [6]. In 2019, Zhang and Fand synthesized all kinds of ZnSe nanostructures by surfactant assisted electrodeposition, including nanorods, nano-spheres, and nano-syringes, which all exist $\sim 2.2 \mathrm{eV}$ deep level emission peak in their PL spectra [7]. During this period, a lot of work was devoted to improving the ratio of near-band edge luminous intensity of ZnSe nanostructures. Although the research of $\mathrm{ZnSe}$ nanostructures has gone through nearly thirty years, many researchers are still very concerned about its research. In recent years, the development of blue light devices based on $\mathrm{ZnSe}$ nanostructures is also in full swing. For example, Ou et al. studied novel blue-light-emitting diodes based on nanostructured $\mathrm{ZnSe} / \mathrm{ZnS}$ multilayer films [8] and Wisniewski et al. developed based on ZnSe nanowire field effect transistors [3]. Therefore, blue light applications based on ZnSe nanostructures have very broad prospects.

In the paper, a simple chemical vapor deposition (CVD) method assisted by tin dioxide $\left(\mathrm{SnO}_{2}\right)$ is proposed to prepare $\mathrm{ZnSe}$ nanowires. Adding a small amount of $\mathrm{SnO}_{2}$ powder to the $\mathrm{ZnSe}$ source powder can directly grow ZnSe nanowires with only near band edge emission by the CVD method. During the growth process, $\mathrm{SnO}_{2}$ powder can not only be used as a catalyst to reduce the growth temperature of nanowires, but also can enhance the near-band edge luminescence of ZnSe nanostructures, thereby avoiding deep-level defect emission peaks.

\section{Experimental Method}

ZnSe nanowires were synthesized by a one-step CVD method. High-purity $\mathrm{ZnSe}$ and $\mathrm{SnO}_{2}$ mixed powders with mass ratio 20:1, load in a ceramic boat, were placed in the center of the horizontal tube furnace. Silicon substrates were located about $10 \mathrm{~cm}$ downstream away from the center of the tube furnace to receive the grown $\mathrm{ZnSe}$ nanowires. The tube furnace is supplied with $\operatorname{Ar}(90 \%) / \mathrm{H}_{2}(10 \%)$ carrier gas to purify the growth atmosphere of nanowires in the tube furnace. Then the mixed powders placed in the middle of the tube furnace were heated at the rate of $120^{\circ} \mathrm{C} / \mathrm{min}$ to $1050^{\circ} \mathrm{C}$ and hold for 30 minutes at this temperature for nanowires growth. When the deposition finished and furnace was naturally cooled to room temperature, yellow colored $\mathrm{ZnSe}$ nanowires can be obtained on silicon wafers.

The morphology of the nanowires was characterized by SEM (Zeiss SUPRA 55) equipped with EDS. Photoluminescence (PL) spectra were carried out by a 
laser cofocal photoluminescence microscope system (Princeton Instruments Acton SP2500) using continuous wavelength $405 \mathrm{~nm}$ laser as the excitation source. Temperature-dependent PL spectra $(77-300 \mathrm{~K})$ were measured by liquid-nitrogen-cooled cryostat system (Janis ST-500, Microscope Cryostat).

\section{Results and Discussion}

\subsection{SEM Analysis}

Figure 1 shows the morphology and element content analysis of ZnSe nanowire. Figure 1(a) is an SEM photograph of the nanowire. It can be seen from Figure 1 (a) that there is a large ball drop at the top of the nanowire, which is a sign of the gas-liquid-solid growth process. Figure 1(b) and Figure 1(c) are the analysis of the element content at position S1 and S2 in Figure 1(a), respectively. It can be seen from Figure 1(b) that the main elements at position $S 1$ of the nanowire are $\mathrm{Zn}$ and Se, while they are $\mathrm{Sn}$ and $\mathrm{O}$ at position S2. It indicates that the $\mathrm{SnO}_{2}$ is used as a catalyst to assist the growth of $\mathrm{ZnSe}$ nanowires.

\subsection{Luminescence Properties Analysis}

In order to characterize the luminescence properties of the $\mathrm{ZnSe}$ nanowires, the steady state PL spectra of the nanowires were tested by continuous wave $405 \mathrm{~nm}$ laser. Figure 2 exhibits typically luminescence property of $\mathrm{ZnSe}$ nanowires grown by $\mathrm{SnO}_{2}$-assisted CVD method. The PL spectrum of the nanowire in Figure 2(a) shows that the nanowires only have near band edge emission, and the center of the PL peak is at $475 \mathrm{~nm}$, which is different from the nanowires grown by an Au-catalyzed CVD method [9] [10], and there is no obvious deep-level defect emission peak at the low energy levels of its PL spectrum. During the growth or synthesis of $\mathrm{ZnSe}$ nanostructures, point defects such as $\mathrm{Zn}$ vacancies and $\mathrm{Zn}$ interstitials and structural defects such as dislocations and stacking faults are easily generated, which making their PL spectrum appear strong deep-level luminescence peaks between 500 and $700 \mathrm{~nm}$ [11] [12] [13]. Figure 2(b) is its corresponding emission image in dark field of optical microscope. The spot in
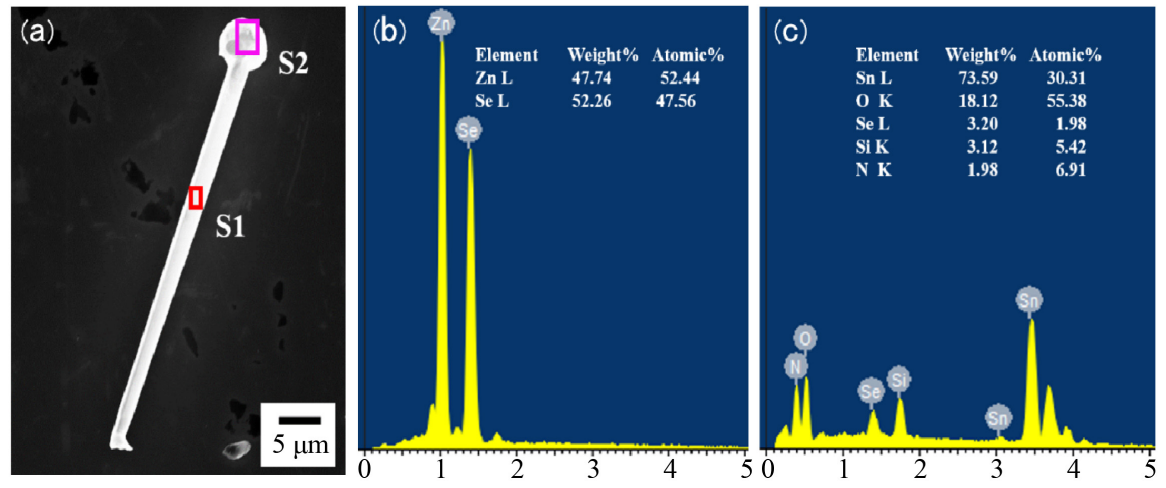

Figure 1. The morphology and element content analysis of ZnSe nanowire. (a) the SEM image of the nanowire; (b) the element content analysis at position S1 of (a); (c) the element content analysis at position S2 of (a). 


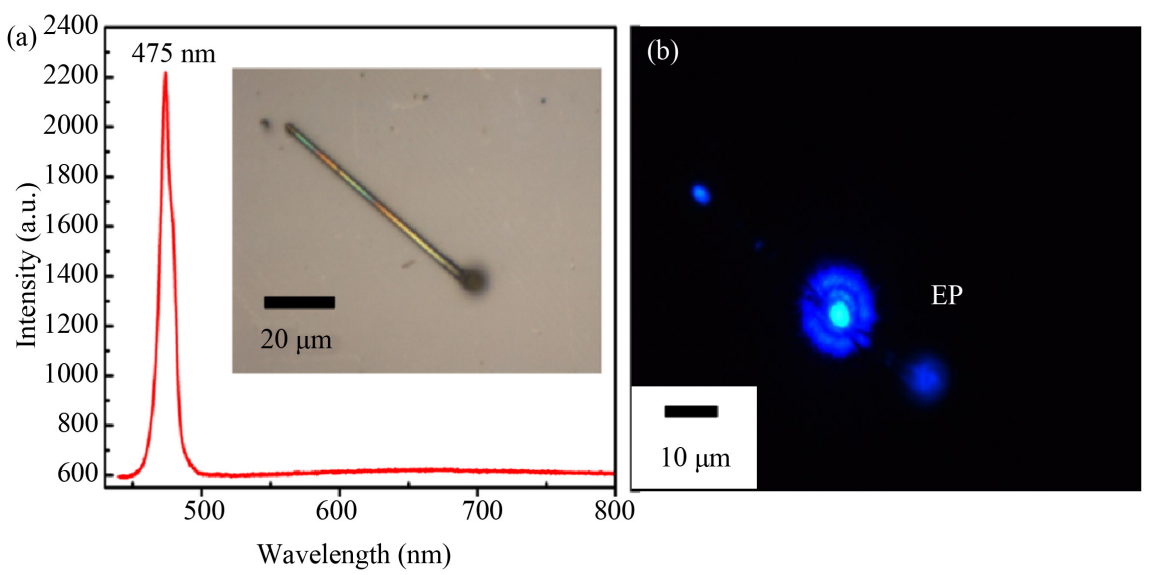

Figure 2. (a) the photoluminescence (PL) spectrum of ZnSe nanowire at room temperature, the inset is the bright-field image of the nanowire in optical microscope. (b) the emission image of nanowire excited by laser in the dark-field of optical microscope.

the middle of the nanowire is the excitation position of the laser, and it can be seen from the image that the nanowire emits blue light.

Figure 3 shows the PL spectra of a single ZnSe nanowire at different excitation positions. Figure 3(a) exhibits its bright-field photo under the microscope, in which the red dots mark the different excitation positions of the laser on the nanowire. The inset in the upper right corner demonstrates the emission image of nanowires in the dark field at the excitation position P5. Figure 3(b) exhibits their corresponding PL spectra. It can be seen from the PL spectra that as the excitation position of the nanowire changes, the emission peak of the nanowire shifts from 473 to $503 \mathrm{~nm}$. The shift of emission peak indicates that there may be defect-related bound state inside the nanowire due to the doping of $\mathrm{SnO}_{2}$, which needs further research.

Figure 4 demonstrates PL spectra of $\mathrm{ZnSe}$ nanowire at different temperature ranging from 77 - $300 \mathrm{~K}$. Figure 4(a) shows the PL spectrum of individual ZnSe nanowire at $77 \mathrm{~K}$, which exhibits blue light emission of nanowire at near band-edge and doesn't exist deep level yellow light emission. The emission peak, center at $456 \mathrm{~nm}$, is assigned to the bound exciton (BX) emission of ZnSe nanowire, Compared with the emission peak of free excitons in ZnSe nanowires [14], the band edge emission energy has shifted to a lower energy level by $24 \mathrm{meV}$. Obviously, there are other bound state emissions at low energy side of BX, as shown in Figure 4(a). Figure 4(b) shows the PL spectra of ZnSe nanowire at different temperatures ranging from $77-300 \mathrm{~K}$. With temperature increasing, near band-edge emission peaks produce large redshift from 456 to $487 \mathrm{~nm}$. This is because as the temperature rises, the nanowire lattice expands, and the electron-phonon coupling interaction is enhanced, which changes the band gap of the ZnSe nanowire, causing the emission peak of BX is red-shifted [15] [16]. Furthermore, the redshift of free excitons does not produce such a large movement [17], which also just proves that the emission peak originates from the emission of bound excitons. Although the content of $\mathrm{Sn}$ or $\mathrm{O}$ is not measured by 

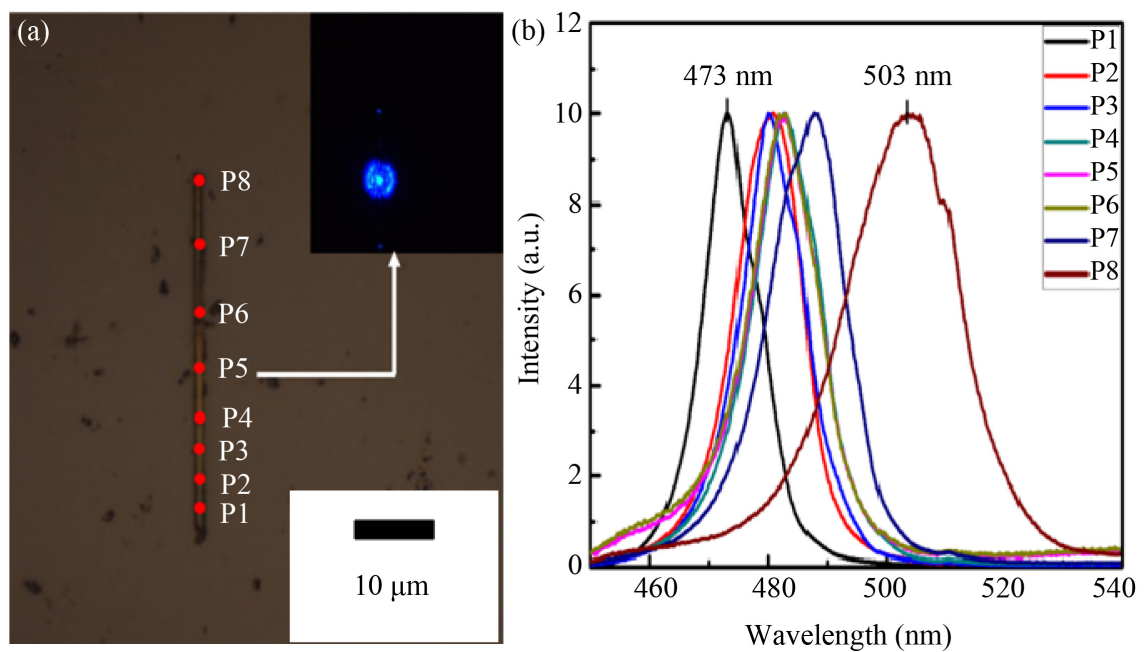

Figure 3. (a) the bright-field image of the ZnSe nanowire in optical microscope, the red spots are corresponding excited positions, the inset is the emission image of nanowire excited at P5 in the dark-field of optical microscope. (b) the PL spectra at different exciting positions of same $\mathrm{ZnSe}$ nanowire at room temperature.
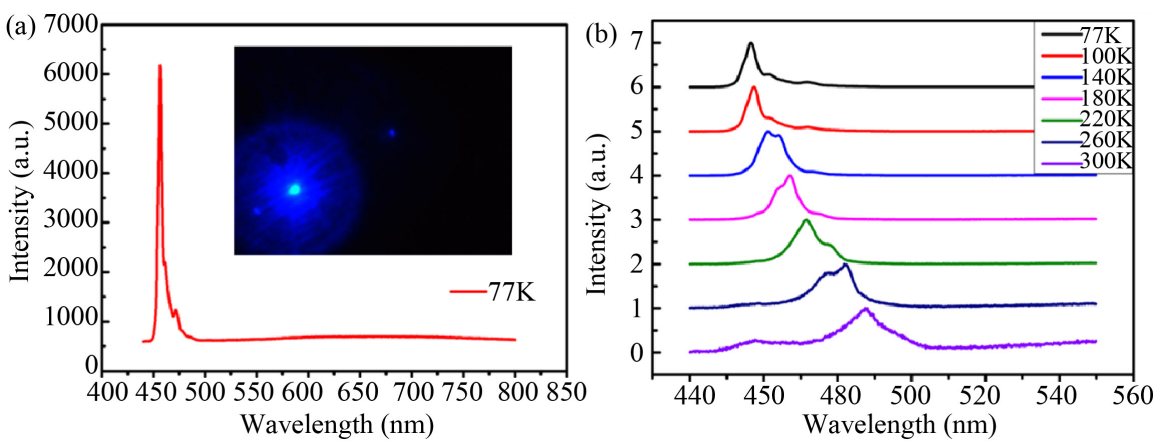

Figure 4. The PL spectra of ZnSe nanowire at different temperature. (a) The PL spectrum at $77 \mathrm{~K}$. The inset is its corresponding emission image. (b) The PL spectra at different temperature ranging from $77-300 \mathrm{~K}$.

EDS, the generation of bound state is related to the doping of $\mathrm{SnO}_{2}$, and their relationship will be further studied in the next work.

\section{Conclusion}

In conclusion, enhanced near band-edge emission $\mathrm{ZnSe}$ nanowires have been grown by $\mathrm{SnO}_{2}$ assisted CVD method. When using the CVD method to grow $\mathrm{ZnSe} \mathrm{nanomaterials,} \mathrm{adding} \mathrm{a} \mathrm{small} \mathrm{amount} \mathrm{of} \mathrm{SnO}_{2}$ to the source material $\mathrm{ZnSe}$ powder can obtain $\mathrm{ZnSe}$ nanomaterials with only blue edge emission. From its optical characterization, it can be found that there is only $475 \mathrm{~nm}$ near-band edge emission peak at room temperature, and no deep-level defect emission. At the same time, it can be seen from its emission spectra that the emission wavelength of nanowires can be shifted to $503 \mathrm{~nm}$ by changing the growth conditions of the $\mathrm{ZnSe}$ nanowires and adjusting the doping of $\mathrm{SnO}_{2}$. The $\mathrm{ZnSe}$ nanowires obtained by the simple one-step method can improve its application in blue 
light-emitting devices, and solve the problem that the blue light emission of ZnSe nanomaterials is accompanied by strong deep energy level yellow light emission.

\section{Acknowledgements}

This work was supported by Scientific and Technological Innovation Programs of Higher Education Institutions in Shanxi.

\section{Conflicts of Interest}

The authors declare no conflicts of interest regarding the publication of this paper.

\section{References}

[1] Ning, J., Liu, J., Levi-Kalisman, Y., Frenkel, A.I. and Banin, U. (2018) Controlling Anisotropic Growth of Colloidal ZnSe Nanostructures. Journal of the American Chemical Society, 140, 14627-14637. https://doi.org/10.1021/jacs.8b05941

[2] Kim, Y., Im, H.S., Park, K., Kim, J., Ahn, J.-P., Yoo, S.J., Kim, J.-G. and Park, J. (2017) Bent Polytypic ZnSe and CdSe Nanowires Probed by Photoluminescence. Small, 13, Article ID: 1603695. https://doi.org/10.1002/smll.201603695

[3] Wisniewski, D., Byrne, K., de Souza, C.F., Fernandes, C. and Ruda, H.E. (2018) Enhancement of Transport Properties in Single ZnSe Nanowire Field-Effect Transistors. Nanotechnology, 30, Article ID: 054007. https://doi.org/10.1088/1361-6528/aaf0d9

[4] Ishikura, H., Abe, T., Fukuda, N., Kasada, H. and Ando, K. (2000) Stable Avalanche-Photodiode Operation of ZnSe-Based $\mathrm{p}(+)-\mathrm{n}$ Structure Blue-Ultraviolet Photodetectors. Applied Physics Letters, 76, 1069-1071.

https://webvpn.bit.edu.cn/https/77726476706e69737468656265737421f1fe51d23433 61447f1c80a3961b2c275c/doi/10.1063/1.125941 https://doi.org/10.1063/1.125941

[5] Liang, Y., Tao, Y. and Hark, S.K. (2011) Fabrication and Optical Properties of Vertically Aligned ZnSe Nanowire Arrays Catalyzed by Ga and Ag. CrystEngComm, 13, 5751-5754. https://doi.org/10.1039/c1ce05412k

[6] Zannier, V., Martelli, F., Grillo, V., Plaisier, J.R., Lausi, A. and Rubini, S. (2014) Strong Blue Emission from ZnSe Nanowires Grown at Low Temperature. Physica Status Solidi (RRL) - Rapid Research Letters, 8, 182-186. https://doi.org/10.1002/pssr.201308264

[7] Zhang, H. and Fang, Y. (2019) Temperature Dependent Photoluminescence of Surfactant Assisted Electrochemically Synthesized ZnSe Nanostructures. Journal of Alloys and Compounds, 781, 201-208. https://doi.org/10.1016/j.jallcom.2018.11.375

[8] Ou, K., Wang, S., Zhang, X. and Yi, L. (2019) Novel Blue-Light-Emitting Diodes Based on Nanostructured ZnSe/ZnS Multilayer Films. Journal of Materials Science, 54, 4049-4055. https://link.springer.com/article/10.1007/s10853-018-3132-4 https://doi.org/10.1007/s10853-018-3132-4

[9] Hou, L.P., et al. (2018) Bound Magnetic Polaron in Zn-Rich Cobalt-Doped ZnSe Nanowires. Nanotechnology, 29, Article ID: 055707.

http://stacks.iop.org/0957-4484/29/i=5/a=055707 https://doi.org/10.1088/1361-6528/aaalbe 
[10] Zou, Y., Li, H., Ren, P., Xu, J., Ma, L., Wang, X., Fan, X., Shan, Z., Zhuang, X., Zhou, H., Zhu, X., Zhang, Q. and Pan, A. (2014) Microphotoluminescence of Individual ZnSe Nanoribbons. Materials Letters, 129, 118-121.

https://doi.org/10.1016/j.matlet.2014.05.015

[11] Philipose, U., Xu, T., Yang, S., Sun, P., Ruda, H.E., Wang, Y.Q. and Kavanagh, K.L. (2006) Enhancement of Band Edge Luminescence in ZnSe Nanowires. Journal of Applied Physics, 100, Article ID: 084316. https://doi.org/10.1063/1.2362930 http://scitation.aip.org/content/aip/journal/jap/100/8/10.1063/1.2362930

[12] Xing, G., Luo, J., Li, H., Wu, B., Liu, X., Huan, C.H.A., Fan, H.J. and Sum, T.C. (2013) Ultrafast Exciton Dynamics and Two-Photon Pumped Lasing from ZnSe Nanowires. Advanced Optical Materials, 1, 319-326.

https://doi.org/10.1002/adom.201200045

[13] Zhang, X.T., Liu, Z., Leung, Y.P., Li, Q. and Hark, S.K. (2003) Growth and Luminescence of Zinc-Blende-Structured ZnSe Nanowires by Metal-Organic Chemical Vapor Deposition. Applied Physics Letters, 83, 5533-5535. https://doi.org/10.1063/1.1638633

[14] Hite, G.E., Marple, D.T.F., Aven, M. and Segall, B. (1967) Excitons and the Absorption Edge in ZnSe. Physical Review, 156, 850-859.

https://journals.aps.org/pr/abstract/10.1103/PhysRev.156.850 https://doi.org/10.1103/PhysRev.156.850

[15] Varshni, Y.P. (1967) Temperature Dependence of the Energy Gap in Semiconductors. Physica, 34, 149-154. https://doi.org/10.1016/0031-8914(67)90062-6

[16] Liu, B., Chen, R., Xu, X.L., Li, D.H., Zhao, Y.Y., Shen, Z.X., Xiong, Q.H. and Sun, H.D. (2011) Exciton-Related Photoluminescence and Lasing in CdS Nanobelts. The Journal of Physical Chemistry C, 115, 12826-12830. https://doi.org/10.1021/jp203551f

[17] Hou, L., Zhou, W., Zou, B., Zhang, Y., Han, J., Yang, X., Gong, Z., Li, J., Xie, S. and Shi, L.-J. (2017) Spin-Exciton Interaction and Related Micro-Photoluminescence Spectra of ZnSe: Mn DMS Nanoribbon. Nanotechnology, 28, Article ID: 105202. https://www.ncbi.nlm.nih.gov/pubmed/28077805 https://doi.org/10.1088/1361-6528/aa58f1 